Harold A. McAlister and William I. Hartkopf (eds.)

\title{
A Two-Dimensional Near-Infrared Speckle Imaging Survey of T Tauri Stars in Taurus and Ophiuchus
}

\author{
A. GHEZ, G. NEUGEBAUER, \& K. MATTHEWS \\ Palomar Observatory, California Institute of Technology 320-47, \\ Pasadena, CA 91125, USA
}

\begin{abstract}
We present the results of a magnitude limited (K $\leq 8.5 \mathrm{mag})$ multiplicity survey of $T$ Tauri stars in the two nearest star forming regions, Taurus-Auriga and Ophiuchus-Scorpius ( $D=150 \mathrm{pc}$ ), observable from the northern hemisphere. Each of the 70 stars in the sample was imaged at $2.2 \mu \mathrm{m}$ using two-dimensional speckle interferometry resulting in a survey sensitive to binary stars with separations ranging from $0^{\prime \prime} .09$ to about $2^{\prime \prime} .5$.

The frequency of double stars with separation in this range is $46 \pm 8 \%$. A division between the classical $T$ Tauri stars and the weak-lined $T$ Tauri stars shows no distinction. Furthermore, no difference is observed between the binary frequencies in the two star forming regions although the clouds have very different properties.

Given the limited angular separation range that this survey is sensitive to, both the spectroscopic and wide binaries will be missed. The rate at which binaries are detected suggests that most, if not all, $\mathrm{T}$ Tauri stars have companions.
\end{abstract}

\section{INTRODUCTION}

$\mathrm{T}$ Tauri stars are a class of young, low mass $\left(0.1\right.$ to $\left.3.0 \mathcal{M}_{\odot}\right)$, pre-main sequence stars whose age is in the range of $10^{4}-10^{7}$ years. Most evolutionary theories of how these stars emerge from the deeply embedded protostellar stage and progress toward the main sequence are based on single star scenarios (e.g., Adams et al. 1988) and yet main sequence surveys have already shown that about $2 / 3$ of solar-type stars are in multiple systems (Abt 1983, Duquennoy \& Mayor 1991). The question arises of how and when these multiple star systems form, for they are clearly the norm as opposed to the exception.

We have carried out a survey of $\mathrm{T}$ Tauri stars located in nearby star forming regions to look for close companions using the high angular resolution technique of speckle imaging. In interpreting the results of this survey, one can compare the multiplicity statistics of (1) T Tauri stars in the two different star forming regions, (2) T Tauri stars with and without evidence of inner accretion disks, and (3) T Tauri stars and solar-type main sequence stars. This paper concentrates on the last comparison in the interest of understanding the multiplicity as a function of age. A more complete discussion of this survey will be published elsewhere (Ghez et al. 1992).

\section{TECHNIQUE \& SYSTEM}

Speckle imaging is a technique used to recover diffraction-limited images from data obtained in the presence of atmospheric turbulence. The details of the data analysis are not given here, but can be found in Ghez et al. (1990).

The data for this survey were obtained at the $f / 15$ Cassegrain focus of the Hale 5-m telescope on Palomar Mountain using a $58 \times 62 \mathrm{InSb}$ array camera in 


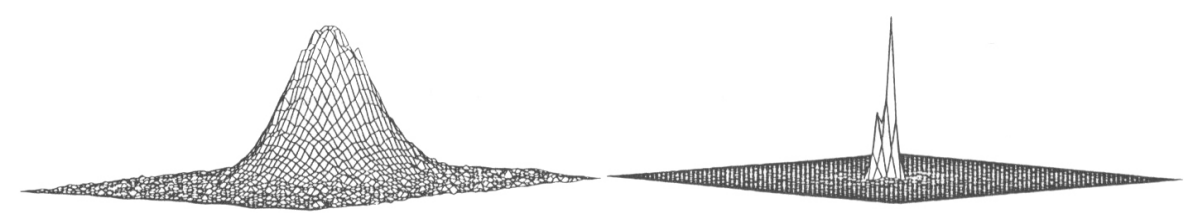

FIGURE 1. The direct image (left) of V773 Tau at $2.2 \mu \mathrm{m}$ with resolution limited to $1^{\prime \prime} .0$ by atmospheric turbulence. The speckle image (right) of the same source reveals the presence of a binary star with separation $0^{\prime \prime} .11$.

the photometric $\mathrm{K}(2.2 \mu \mathrm{m})$ band. The pixel scale, determined by observing and reconstructing images of several binaries with well-known orbits (McAlister \& Hartkopf 1988), is $0 \prime .053 \times 0^{\prime \prime} .053\left( \pm 0^{\prime \prime} .001\right)$. This system is sensitive to binary stars with separations between $0^{\prime \prime} .09$ to about $2^{\prime \prime} .5$, where the lower limit is set by the diffraction limit of the telescope and the upper limit is set by the field of view of the array. Since each target star is placed roughly in the center of the detector, companions outside half the field of view will not be detected. The detector is not circular so the exact outer limit depends on position angle and thus is not sharply defined.

An example of the spatial resolution gained by using speckle imaging is shown in Figure 1.

\section{SURVEY SAMPLE}

The survey was confined to the two nearest star forming regions observable from the northern hemisphere, Taurus-Auriga (140 pc, Elias 1978b) and OphiuchusScorpius (160 pc, Elias 1978a). At 150 pc, the average distance to these two regions, the range of angular binary star separations to which this survey is sensitive, $0.09-2 " .5$, corresponds to a projected linear separation range of 13 $375 \mathrm{AU}$.

The sample was compiled by selecting all known $T$ Tauri stars with $K \leq 8.5$ mag (the limiting magnitude of the speckle system used), in the regions of interest. The resources for identifying $\mathrm{T}$ Tauri stars were the optically selected source lists from Herbig \& Bell (1988), and the x-ray selected source lists in Bouvier \& Appenzeller (1991), Walter et al. (1988) and Walter (1992). A total of $146 \mathrm{~T}$ Tauri stars were identified, of which 98 were brighter than the survey's magnitude limit. Over the period 1990 July to 1991 November, 70 of these stars have been observed. 


\section{RESULTS}

Of the 70 target stars observed, 32 were detected as double stars by speckle imaging; 16 of these double stars were previously unreported. These doubles are all assumed to be physically bound since the probability of chance alignment with a field star within $2^{\prime \prime} .5$ of the target is negligible for both regions.

Before calculating a binary frequency for this sample, we need to know whether or not any two target stars are members of a single wide binary system. This will determine how many independent $\mathrm{T}$ Tauri systems were observed. We adopt a cutoff for wide binary star separations of $2 \times 10^{3} \mathrm{AU}$, which corresponds to $13^{\prime \prime}$ at $150 \mathrm{pc}$. Thus targets stars are characterized as primary and secondary: a secondary target star is a target star separated from a brighter (at $2.2 \mu \mathrm{m}$ ) target star by less than $13^{\prime \prime}$, but by more than about $2^{\prime \prime} .5$ (i.e. it was observed separately). In this sample only one of the $\mathbf{7 0}$ target stars was considered to be a secondary target star, leaving 69 primary target stars or independent $\mathrm{T}$ Tauri systems.

We define the binary star frequency to be the number of companion stars, within the specified separation range, per the number of systems examined. For this survey the binary star frequency, in the projected linear separation range $13-375 \mathrm{AU}$, is $46 \pm 8 \%$. Table 1 lists the binary star frequency of this sample as a whole as well as dividing it by star forming region and by strength of $H \alpha$ emission (for a discussion of these internal comparisons see Ghez et al. 1992).

TABLE 1. T Tauri Binary Star Frequencies $13-375$ AU

\begin{tabular}{|lccc|}
\hline Sample & $\begin{array}{c}\text { Number of } \\
\text { Systems }\end{array}$ & $\begin{array}{c}\text { Number of } \\
\text { Companions }\end{array}$ & $\begin{array}{c}\text { Binary } \\
\text { Frequency (\%) }\end{array}$ \\
\hline Total & 69 & 32 & $46 \pm 8$ \\
Tau & 46 & 21 & $46 \pm 10$ \\
Oph & 23 & 11 & $48 \pm 14$ \\
WTTS & 24 & 11 & $46 \pm 14$ \\
CTTS & 45 & 21 & $47 \pm 10$ \\
\hline
\end{tabular}

Figure 2 displays the distribution of the $\mathrm{K}(2.2 \mu \mathrm{m})$ flux ratio for all the $\mathrm{T}$ Tauri binary stars in this sample (circles). We have also included the $\mathrm{K}$ flux ratio of $T$ Tauri binary stars detected by other techniques and not necessarily observed in this survey.

The wide binary stars observed with direct imaging techniques (Moneti \& Zinnecker 1991; Simon et al. 1992) appear to reach much higher flux ratios than either those observed here or with lunar occultation (Simon et al. 1992). This could be the result of a number of possible effects; either (1) the wide binary stars with high flux ratios are due to chance projections, (2) the techniques sensitive to the more closely spaced binary stars are not sensitive to the high flux ratio binary stars, or (3) there is an inherent difference between wide and close binary stars with a division at a separation of roughly $100 \mathrm{AU}$. Here we will just note that the observed binary frequency may be a lower limit to the true 


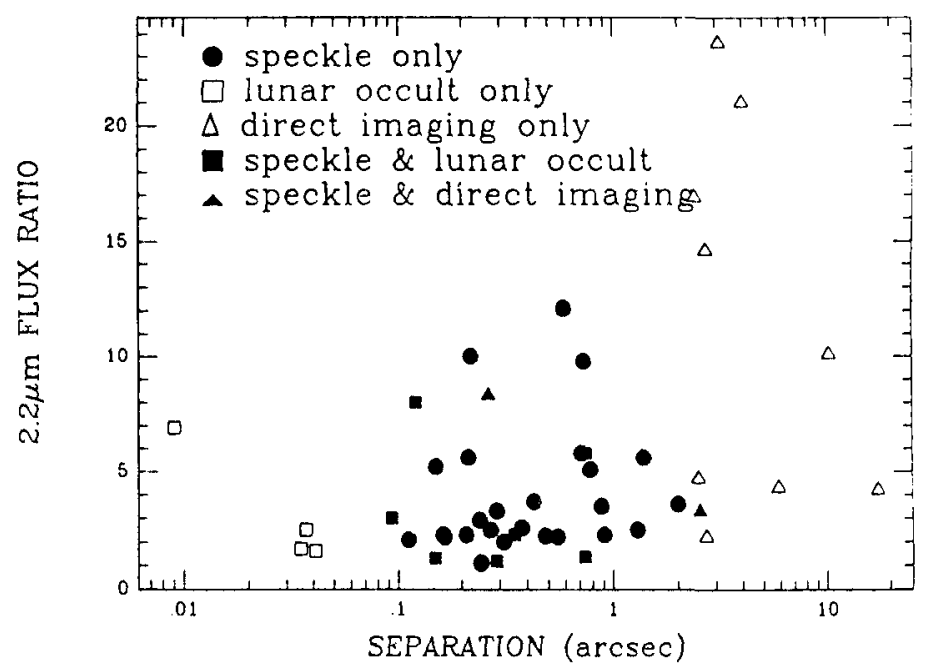

FIGURE 2. The distribution of $\mathrm{K}(2.2 \mu \mathrm{m})$ flux ratios as a function of projected linear separation for all the $T$ Tauri binary stars found in this survey as well as those found with other techniques. The filled points indicate those binary stars that were measured with speckle imaging. The separations given for binary stars measured with lunar occultations alone (the unfilled squares) are one dimensional separations (along the path of the moon).

binary frequency in the projected linear separation range $13-375 \mathrm{AU}$. Limits for individual observations will be given in Ghez et al. (1992).

\section{T TAURI STARS VERSUS SOLAR-TYPE MAIN SEQUENCE STARS}

A comparison of the multiplicity statistics of $\mathrm{T}$ Tauri stars and their older counterparts on the main sequence is useful in studying the question of how and when binary stars form. The comparison that will be made here is between the binary frequency of the $\mathrm{T}$ Tauri sample in this survey and the $\mathrm{G}$ dwarf sample studied by Duquennoy \& Mayor (1991). To make a proper comparison between these two surveys it is necessary to (1) normalize the binary star distribution of the samples consistently and (2) measure this distribution as a function of the same variable.

Figure 3 shows the binary star frequency as a function of orbital period for the G-dwarf sample (Duquennoy \& Mayor 1991). It is a histogram of all the orbiting pairs of stars in the sample of 164 primary target stars (with a similar definition of primary versus secondary as given above). It should be noted that more than just binary stars are counted as orbiting pairs of stars. The counting of pairs is as follows: one for each double star, two for each triple star, and three for each quadrupole. A correction for detection biases is included in this distribution to account for undetected, mostly visual, pairs. To make the 


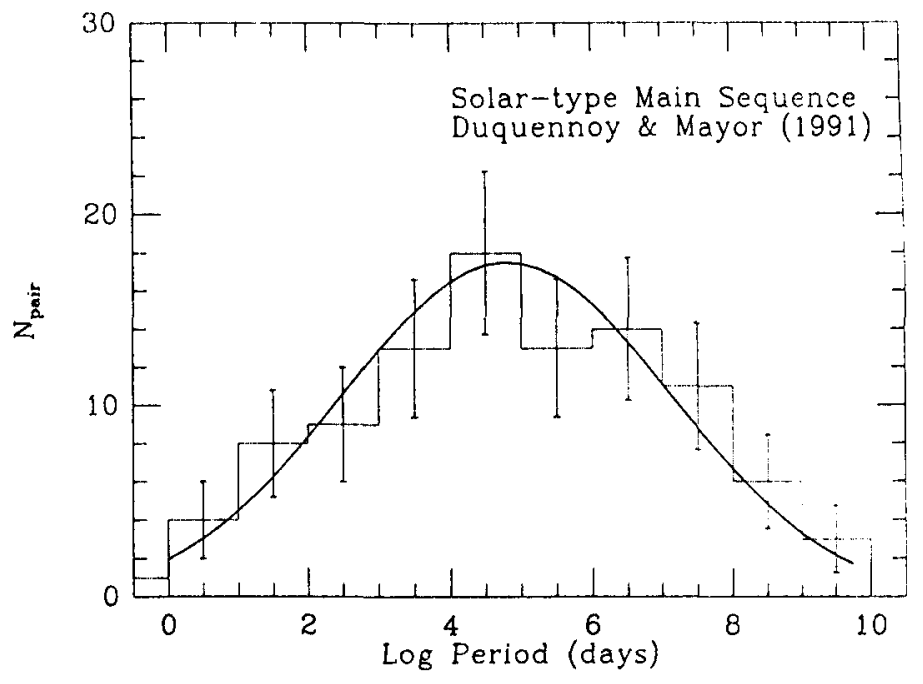

FIGURE 3. A histogram of orbiting pairs of stars in a sample of solar-type main sequence stars, taken from Duquennoy \& Mayor (1991)

comparison with the T Tauri sample, which has a different number of targets, we have normalized this distribution by the number of primary targets. In this way the normalization is independent of unresolved companion stars.

We chose to convert the distribution of solar-type main sequence binary stars as a function of orbital period to a function of projected linear separation, the variable directly deduced in the present survey. This was done in two steps: first, the orbital period was converted to its corresponding semi-major axis by using Kepler's law, with the assumption that each binary system was composed of two $1 \mathcal{M}_{\odot}$ stars. Second, we obtained an average projected separation from the semi-major axis using a monte carlo simulation carried out by Fischer \& Marcy (1992).

The final step taken was to rebin the nearby G-dwarf binary star distribution such that the new bin width matches our separation range, using the Gaussian model fit Duquennoy \& Mayor (1991) made to their original distribution to correct for the difference in bin boundaries. The comparison between the two data sets is shown in Figure 4, where the central bin in the histogram corresponds to the separation range $13-375 \mathrm{AU}$. In this range, $20 \pm 1 \%$ of the solar-type main sequence stars, compared to $46 \pm 8 \%$ of the T Tauri star sample, have companion stars.

This $3.2 \sigma$ discrepancy suggests $\mathrm{T}$ Tauri stars have twice as many companions as compared to solar-type main sequence stars in the projected linear separation range $13-375 \mathrm{AU}$.

If this discrepancy is real, it can be interpreted in a number of ways. One must, however, treat any interpretation of a $3.2 \sigma$ discrepancy with a great deal of caution. We have considered two possible explanations for an over abundance of companion stars amongst the $\mathrm{T}$ Tauri stars relative to the nearby G-dwarf 


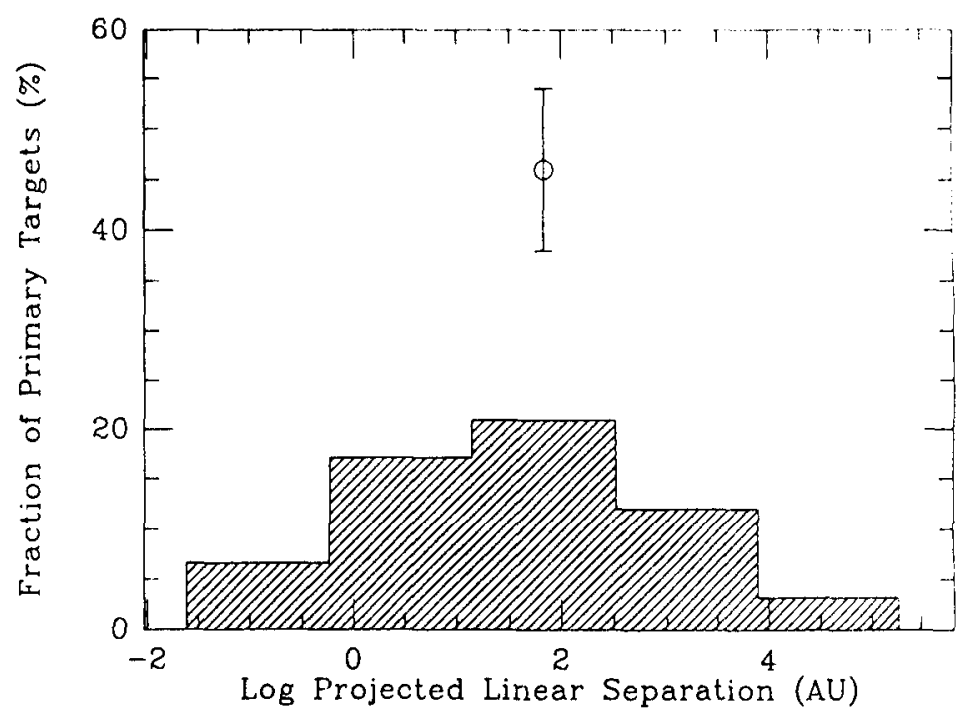

FIGURE 4. Comparison of the binary star distribution for the solar-type main sequence stars (hatched histogram) with the T Tauri stars (the circle which corresponds to the central histogram bin). The T Tauri stars appear to have twice as many companions between $13-375 \mathrm{AU}$, projected linear separation, at the $3.2 \sigma$ level.

stars in the projected linear separation range 13 - $375 \mathrm{AU}$; (1) a difference in survey sensitivity and (2) an intrinsic difference in the properties of the two samples.

If the difference is due to a difference in the sensitivity of the two surveys, that would imply that our survey is much more sensitive to companion stars than Duquennoy \& Mayor's (1991) survey in the projected linear separation range $13-375 \mathrm{AU}$. However, Figure 2 has already shown that speckle imaging has not observed particularly high flux ratio binary stars relative to direct imaging results. Furthermore, the G-dwarf sample has already been corrected for undetected companion stars. Thus it seems unlikely that apparent overabundance of companion stars amongst the T Tauri stars compared to the G-dwarf stars is the result unaccounted companions in Duquennoy \& Mayor's sample.

If the difference in the binary star frequency between the $T$ Tauri stars and their older counterparts on the main sequence is due to an intrinsic difference in their properties (i.e. depends on their age), then the stars in the current $T$ Tauri star sample will have the same properties as the G-dwarf sample once they have evolved down to the main sequence. Here we explore two possible evolutionary scenarios that might explain the putative overabundance of companion stars at the younger stage of evolution in the projected linear separation range $13-375$ AU.

First, we will consider the possibility that $\mathrm{T}$ Tauri stars and solar-type main sequence stars have the same number of companion stars integrated over all possible separations. In this case an overabundance of companion stars in the T Tauri sample with respect to the G-dwarf sample in the projected linear 
separation 13 - $375 \mathrm{AU}$ requires a deficiency of companions amongst the $\mathrm{T}$ Tauri stars at separations outside this range so that the integrated number of companion stars would be the same for both samples. Thus the observed difference could result from $T$ Tauri binary star distribution being more centrally peaked, as opposed to a difference in the overall binary frequency. This implies that the distribution of binary stars as a function of separation would spread or relax as a function of time. If this hypothesis is true then integrating the histogram in Figure 4 indicates that a total of 43 pairs should be found in the whole sample of $69 \mathrm{~T}$ Tauri systems. We can explore this possibility by inquiring about other companion stars in the $\mathrm{T}$ Tauri star sample discovered with techniques that are sensitive to different separation ranges compared to speckle imaging. Table 2 lists the current state of overlapping observations with various techniques for the $69 \mathrm{~T}$ Tauri star systems in the survey presented here. The current knowledge of all companion stars in our sample is far from complete, but already brings the total number of companion stars is to 41 and the predicted number is greater than the 43 pairs derived from the assumption that the total number of companion stars does not change as a function of age between the $\mathrm{T}$ Tauri and main sequence stage of evolution. Thus it appears that the total number of companion stars is greater at the $\mathrm{T}$ Tauri stage of evolution.

TABLE 2. Overlap of Sample with Different Techniques

\begin{tabular}{|lcrrr|}
\hline \multicolumn{1}{|c}{ Technique } & Separation Range & $n_{o}^{1}$ & $n_{p}^{2}$ & $N_{p}^{3}$ \\
\hline Direct Imaging ${ }^{a, b}$ & $\geq 2^{\prime \prime} .5-13^{\prime \prime}$ & 27 & 4 & 10 \\
Lunar Occultation $^{a}$ & $0^{\prime \prime} .005-0^{\prime \prime} .09$ & 16 & 2 & 9 \\
Spectroscopy $^{c, d}$ & $0^{\prime \prime} .0002-0^{\prime \prime} .0035$ & 19 & 5 & 6 \\
Speckle $^{\text {s }}$ & $0^{\prime \prime} .09-2^{\prime \prime} .5$ & 69 & 32 & 32 \\
All $^{a, b, c, d, e}$ & $0^{\prime \prime} .0002-13^{\prime \prime}$ & - & 43 & 57 \\
\hline
\end{tabular}

$1 n_{0}$ is the number of stars in present sample observed with a given technique

$2 n_{p}$ is the number of pairs found in the $n_{o}$ stars observed in separation range listed

$3 N_{p}$ is the number of pairs predicted if all of present sample observed, $N_{P}=\frac{69}{n_{0} \times n_{p}}$

a Simon et al. (1992)

- Simon (1992)

c Mathieu et al. (1988)

d Mathieu (1992), we also assume only WTTS will have companion stars

- This work

If there is an overabundance of companions stars integrated over all separations amongst the $T$ Tauri stars as compared to the solar-type main sequence stars, then some of the current $\mathrm{T}$ Tauri star pairs must be disrupted by the time they evolve to the main sequence. One possible mechanism is the disruption of young triple systems. At this point is is worth stressing the importance of observing the same sample with many different techniques. This is necessary to determine the frequency of double, triple, and quadrupole systems, because the various companion stars will not all be observed with any one technique. The 
incomplete overlap of our sample with other techniques has already revealed that $11 \%$ of our sample are members of triple systems whereas Duquennoy \& Mayor (1991) find only $4 \%$ for the solar-type main sequence stars. Thus if the $3.2 \sigma$ discrepancy between the number of companion stars observed for the $T$ Tauri stars and the nearby G-dwarfs is real, unstable triple system may provide a satisfactory evolutionary scenario.

\section{CONCLUSIONS}

We have observed $70 \mathrm{~T}$ Tauri stars in the nearby star forming regions of TaurusAuriga and Ophiuchus-Scorpius using speckle imaging techniques to look for close companion stars. The resulting binary frequency is $46 \pm 8 \%$ in the projected linear separation range $13-375 \mathrm{AU}$.

Compared to Duquennoy \& Mayor's (1991) survey of nearby G-dwarfs, T Tauri stars appear to have twice as many companion stars with projected linear separations in range $13-375 \mathrm{AU}$ at the $3.2 \sigma$ level.

In order to determine the complete $T$ Tauri binary star distribution as a function of separation (or equivalently orbital period) it is necessary to observe the same sample of stars with many different techniques.

\section{REFERENCES}

Abt, H., 1983, ARA $8 A, 21,343$

Adams, F., Lada, C., \& Shu, F. 1987, ApJ, 312, 788

Bouvier, J. \& Appenzeller, I. 1991, $A \& A S$, preprint

Duquennoy, A. \& Mayor, M. 1991, $A \mathcal{E} A, 248,485$

Elias, J.H. 1978a, $A p J, 224,453$

Elias, J.H. 1978b, ApJ, 224, 857

Fischer, D., \& Marcy, J. 1992, submitted

Ghez, A.M., Gorham, P.W., Haniff, C.A., Kulkarni, S.R., Matthews, K., Neugebauer, G., \& Weir, N. 1990, Proc. SPIE, 1237, 249

Ghez, A.M., Neugebauer, G., \& Matthews, K. 1992, in preparation

Herbig, G.H., \& Robbin-Bell, K. 1988, Lick Obs Bull, 1111

Mathieu, R.D., Walter, F. M., \& Myers, P. C. 1989, AJ, 987, 98

Mathieu, R.D. 1992, private communication

McAlister, H.A. \& Hartkopf, W.I. 1988, Second Catalog of Interferometric Measurements of Binary Stars, (Center for High Angular Resolution Astronomy)

Moneti, A. \& Zinnecker, H. 1991, A\&A, 242, 428

Simon, M., Chen, W.P., Howell, R.R., Benson, J.A., \& Slowik, D. 1992, ApJ, 384, 212

Simon, M. 1992, private communication

Walter, F.M., Brown, A., Mathieu, R.D., Myers, P.C., \& Vrba, F.J. 1988, AJ, 96, 297

Walter, F. 1992, private communication

\section{DISCUSSION}

ABT: I did not understand whether you used the same bin width as Duquennoy \& Mayor.

GHEZ: I used a slightly different bin width. It is a bit more than two of their bins, but I used their Gaussian fit to their data to rebin their data to make an 
approproate comparison with my data set.

SIMON: I'd like to comment on the Beckwith et al. $(1990, A J, 99,924) M_{\text {disk }}$ vs. binary separation. You've shown, by adding new speckle binaries, that the correlation is significantly degraded.

MATHIEU: How does the detection sensitivity of $2 \mu \mathrm{m}$ speckle compare with that of visual binaries, from which the main-sequence binary frequency (to which you compare) derives?

GHEZ: I don't think the $2 \mu \mathrm{m}$ measurements are more sensitive. The main sequence pair distribution has been corrected for "missed" binaries.

CHEN: The survey was done at $\mathrm{K}$ band. Have you observed the resolved systems at other bands?

GHEZ: We're beginning to do follow-up measurements.

McALISTER: We published a speckle survey of bright stars (1987, $A J, \mathbf{0 3}$, 183 ) in which we found a significant excess of new binaries at separations closer than $0^{\prime \prime} .25$ over what had previously been found by visual observers. I don't know if our statistics are included in your analysis, and, of course, our results are for earlier type stars.

GHEZ: I didn't include them, but I'll take a look at that paper. Clearly, it's important to understand if there is a difference between early and late type stars.

ABT: I wonder whether the difference in frequencies between you and Duquennoy and Mayor is due simply to your having a higher detection sensitivity?

GHEZ: Possibly, but I would not have expected that to be true since Duquennoy \& Mayor tried to correct for missed companions.

MAZEH: What was the flux ratio limit? Did you compare this limit to the visual data included in the Duquennoy \& Mayor study?

GHEZ: The flux ratio limit depends on the total flux and the seeing conditions. Under good conditions our magnitude limit of $\mathrm{K}=+8.5 \mathrm{mag}$ corresponds to being able to detect a 10-to-1 binary, and this limit increases (i.e. we will see higher flux ratios) with brighter sources. The comparison with the visual data is not straightforward since the T Tauri stars tend to have IR excesses, but this is an important point.

ISOBE: We detected triple star systems for spectroscopic binary stars with separations of several tens of $\mathrm{AU}$ and found the same enhancement of number in this distance range as you detected for $T$ Tauri stars.

GHEZ: What was your sample, and was there a distinction between early and late type stars?

ISOBE: We observed main sequence stars without spectral type distinction.

GHEZ: Perhaps there are more main sequence binary stars than we previously thought, although I think it's more important to separate out the results for solar-type stars. 